\title{
DESARROLLO DE POLÍTICAS DE INTERVENCIÓN URBANA. CASO PROMEBA EN MAR DEL PLATA
}

\section{DEVELOPMENT OF URBAN INTERVENTION POLICIES. PROMEBA CASE IN MAR DEL PLATA}

\author{
Sofía Ariadna González ${ }^{*}$ \\ sofimdq.19@gmail.com \\ ${ }^{1}$ Universidad Nacional de Mar del Plata, Buenos Aires, Argentina \\ *Correspondencia: Sofía Ariadna González. Email: sofimdq.19@gmail.com
}

Recibido: 10.07.21 | Aprobado: 17.08.21

\section{RESUMEN}

La problemática de la informalidad y la conformación de los asentamientos precarios por el desarrollo de la urbanización ha sido objeto de la implementación de un conjunto de políticas sociales y urbanas que buscan paliar los conflictivos procesos de creciente exclusión producto de las políticas neoliberales. En este contexto, este artículo, desde un enfoque cualitativo, indaga en la formación de intervenciones estatales urbanas y sus posibles alcances. Para ello, toma como estudio de caso la implementación del Programa de Mejoramiento de Barrios (ProMeBa) en barrios segregados socioespacialmente en la ciudad de Mar del Plata, lo que permitirá dar cuenta de la urgencia y el posible impacto de las diversas intervenciones de enfoque urbano, así como de la necesidad de adoptar un análisis más integral y holístico de las políticas urbanas.

Palabras clave: Política urbana, condiciones habitacionales, segregación socioespacial, programa de mejoramiento de barrios.

\begin{abstract}
The problem of informality and the formation of precarious settlements due to the development of urbanization has been the object of the implementation of a set of social and urban policies that seek to alleviate the conflictive processes of increasing exclusion resulting from neoliberal policies. In this context, this article, from a qualitative approach, investigates the formation of urban state interventions and their possible scope. To do this, it takes as a case study the implementation of the Neighborhood Improvement Program (ProMeBa) in socio-spatially segregated neighborhoods in the city of Mar del Plata, which will allow to account for the urgency and possible impact of the various interventions of urban approach, as well as the need to adopt a more comprehensive and holistic analysis of urban policies.

Keywords: Urban policy, housing conditions, socio-spatial segregation, neighborhood Improvement Program.
\end{abstract}




\section{INTRODUCCIÓN}

Los procesos de urbanización entre las décadas del '50 y '70, y su profundización en los años '90, han sembrado como característica distintiva en las ciudades de América Latina las grandes extensiones de asentamientos segregados donde el patrón emergente para la organización de las diferencias sociales dentro del espacio urbano se debe a una intensificación espacial del privilegio y la pobreza (Massey, 1996).

En vistas a atender la demanda habitacional, se intensificaron las intervenciones estatales poniéndose en marcha diversos programas e iniciativas urbanas. A diferencia de la estrategia descentralizadora de la década de 1990, desde una nueva gestión estatal se privilegió la centralización, redefiniendo las relaciones del Estado Nacional con Provincias y Municipios, generando nuevas mediaciones masivas apuntadas a viabilizar el acceso al suelo y la vivienda para sectores de menores ingresos. Las mismas, además, pretendieron atender las necesidades de empleo y de mejoras de los aspectos urbano-ambientales de los espacios urbanos segregados.

A partir de lo señalado, esta propuesta propone analizar el desarrollo de políticas de intervención urbana, enfocándose principalmente en el estudio de caso de la implementación del ProMeBa en dos barrios segregados de la ciudad de Mar del Plata.

Para ello, en primera instancia, se describen los conceptos de espacio urbano y de distribución de los agentes sociales sobre el mismo, que tiene como resultado la reproducción de mecanismos de segregación socioespacial. Luego, a través de un recorrido histórico, se analiza el surgimiento de los asentamientos precarios y la formación y desarrollo de políticas de intervención urbana. Por último, se puntualiza en el caso del ProMeBa como política integral de intervención urbana implementada en la ciudad de Mar del Plata a modo de englobar el análisis general.

\section{LA REPRODUCCIÓN DE MECANISMOS DE SEGREGACIÓN SOCIOESPACIAL EN EL ESPACIO URBANO.}

El espacio, en términos burdiesianos, se constituye por la forma en que los agentes o los grupos se distribuyen en él en virtud de la toma de posiciones que hacen dentro del espacio social (Bourdieu, 1997). Topalov, ve el origen de las ciudades en la división social del trabajo y en las relaciones capitalistas entre las fuerzas productivas que la componen. La búsqueda del capital de generar una sobre ganancia y la concentración espacial de los capitales dentro del espacio urbano, obstaculiza la formación de condiciones espaciales de la ciudad. Esto lleva al desarrollo desigual del espacio donde las zonas que no otorgan al capital las condiciones generales de su valoración serán marginadas (Topalov, 1997).

El espacio social reificado, físicamente realizado u objetivado, se presenta entonces como consecuencia de la distribución en el espacio físico de diferentes especies de bienes, agentes individuales y grupos localizados físicamente y provistos de oportunidades más o menos importantes de apropiación de esos bienes y servicios, ya sea en función de su capital y también de la distancia física con respecto a esos bienes. En la relación entre la distribución de los agentes y la distribución de los bienes en el espacio se define el valor de las diferentes regiones del espacio social reificado (Bourdieu, 1997). 
Siguiendo esta línea de pensamiento y desde la discusión regional, Pedro Pírez también invita a pensar a la ciudad como un producto de la relación sociedad-territorio, poniendo el foco en los actores locales como determinantes de los procesos locales en función de la lógica local y su ubicación en el sistema local de relaciones (Pírez, 1995).

La ciudad, por lo tanto, adquiere un valor en función de su acceso a oportunidades económicas y al goce o disposición de ciertos bienes y servicios. Es por esto, que los procesos de urbanización implican la tendencia a ocupación y valorización de aquellas áreas cuya localización resulta más privilegiada en estos términos. Esto lleva a un crecimiento del radio urbano que pocas veces va acompañado de un correspondiente desarrollo de la infraestructura urbana (Oszlak, 1983). En este sentido, autores como Castells (1974) y Duhau (2003), entienden por segregación urbana la tendencia a la organización del espacio en zonas de fuerte homogeneidad social interna y de fuerte disparidad social entre ellas, concibiendo esta disparidad no sólo en términos de diferencia, sino de jerarquía.

Podemos hablar de segregación cuando existe una intención de discriminación hacia un grupo social que se agrega a una situación de fuertes separaciones sociales en el espacio (Brun, 1994). Cuando una ciudad o un espacio se encuentran segregados es porque se forma por partes y no fragmentos, que mantienen interrelaciones de complementariedad y de oposición que diferencian socialmente el espacio, generando un tipo de segregación socio-espacial (Caprón, 2006). Se pueden diferenciar tres dimensiones en la segregación: el grado de concentración espacial de los grupos sociales; la homogeneidad social que presentan las distintas áreas internas de las ciudades; y el prestigio, o desprestigio, social de las distintas áreas o barrios de cada ciudad.

Si bien en la actualidad nos encontramos con mayor crecimiento económico, expansión urbana y mejores condiciones tecnológicas para diseñar el espacio urbano, este escenario lejos está de garantizar otros derechos, como la libertad individual de acceder a los recursos naturales y urbanos que la ciudad ofrece o el reconocimiento de ciudadanía, planteado por Lefebvre (1968) en su concepción del "derecho a la ciudad". Esto se debe a los mecanismos de segregación socio-espacial, el empobrecimiento de la experiencia urbana y la restricción de la participación y democracia urbana que dificultan el acceso a la ciudad a la mayoría de sus habitantes (Do Rio Caldeira, 2007), y que encuentra su expresión en la profunda crisis habitacional materializada en la creciente formación de villas y asentamientos precarios.

\section{EL SURGIMIENTO DE LOS ASENTAMIENTOS PRECARIOS}

El empobrecimiento de la población de los países latinoamericanos es una problemática que se ha ido agravando en las últimas décadas. Durante los años '80, y a partir de los procesos de globalización, el enfoque de la economía de estos países propiciaba principalmente el comercio internacional. Las políticas implementadas en este período generaron un cambio de lógica donde el Estado perdió legitimidad dando paso al mercado competitivo, erigido con las capacidades necesarias para establecer el orden social (Rodríguez, 2009). 
Esta reducción del papel activo del Estado se profundizó en la década de 1990, acentuando aún más la figura del mercado, que se encontraba habilitado a operar libremente (Rodríguez, 2009). Durante este período, los procesos de descentralización y privatización surgieron como nuevos paradigmas de desarrollo, a la par que se produjo un aumento profundo de la pobreza, y en consecuencia, de los procesos de informalidad urbana. Este escenario, llevó a desplegar nuevas estrategias de acceso al suelo y la vivienda, impulsando el desarrollo de autoconstrucción del hábitat vulnerable e inseguro en villas y asentamientos precarios (Clichevsky, 2003; Fernandes, 2003).

En el caso de Argentina, el país se encontraba en un proceso de reforma estructural, donde se alentaban las privatizaciones, el ajuste fiscal, la concentración y centralización de capital, la apertura de comercio, la desregulación y descentralización, el desempleo, la reducción de cargas sociales, la descentralización por tiempo indeterminado, el endeudamiento y la exclusión social (Notcheff, 1999; Rodríguez, 2009). Este escenario, propició nuevas técnicas de producción del hábitat, a partir de la cual empezaron a generarse nuevos procesos de tomas de tierra y organización de demandas urbanas, los llamados asentamientos precarios.

A diferencia de las villas, las cuales habían comenzado a configurarse en la década de 1930, estas tomas colectivas se realizaron con trazados urbanos, regulares y planificados, y se percibieron como una solución habitacional tendiente a presentar mejoras y no como algo transitorio del momento (Merklen; 1991, Cravino; 1998). Los asentamientos precarios, se caracterizan por instalarse en zonas alejadas y sin recursos urbanos, constituyéndose como espacios segregados que llevan a que sus habitantes se encuentren en una situación de vulnerabilidad y desprotección, con condiciones de precariedad habitacional y sin reconocimiento formal y jurídico alguno sobre el espacio urbano que ocupan (Rodríguez, 2009).

Luego de la crisis del 2001, comienza a reactivarse la economía nacional, en parte por la expansión de la demanda de suelo para diversos proyectos nuevos, y en correlación, del sector de la construcción, lo que impulsó fuertemente la actividad del mercado inmobiliario (CELS, 2015; Canestraro, 2016). Esto trajo a consecuencia no sólo un incremento de la inquilinización, sino también, un considerable aumento de la exclusión percibida en la densificación poblacional en villas y asentamientos precarios, en tanto únicos espacios habitables accesibles para muchas familias (Kessler, 2014). De este modo, el acceso al suelo urbano deja de verse únicamente como un componente decisivo en lo que respecta la integración a la ciudad y el reconocimiento de ciudadanía, sino también como un elemento reproductor de prácticas de segregación social y espacial (DurandLasserve, 1997).

La generación de áreas espacialmente segregadas o "informales"(en relación a la complejidad del concepto y las controversias existentes en torno al debate "formalidadinformalidad" puede verse Canestraro, 2013), donde el mercado es quien decide a dónde deben ubicarse los distintos sectores sociales, han puesto de manifiesto el efecto de esta segregación en las condiciones de vida de las personas que en ellos habitan y en la configuración de ciudadanías restringidas basadas en accesos diferenciales a los bienes y 
servicios básicos desde la lógica de mercado de las privatizaciones, desregulaciones y tercerizaciones del espacio urbano.

Precisamente, al hablar de condiciones de vida se hace referencia a una multiplicidad de dimensiones: ambientales, políticas, físicas, culturales, morales y habitacionales, que se vinculan con las esferas políticas, económicas y sociales de las naciones (Scarponetti, 2019). Las condiciones de vida de una población se pueden identificar desde dos dimensiones distintas: desde la pública y la privada. La primera, responde al acceso a infraestructuras colectivas y sostenibilidad del ambiente. La segunda, refiere a especificidades propias de la educación, la vivienda y el empleo (Velazquez, 2007; Canestraro y Elorza, 2019). Por lo tanto, al hablar de condiciones habitacionales en particular no se hace referencia únicamente al acceso democrático al espacio en el que vivir o al lugar donde habitar, sino que incluye también el acceso a un medio habitable, con acondicionamiento físico y social, socialmente generado, que permita el desarrollo de asentamientos sociales (Trivelli, 1982), el acceso a infraestructura y servicios urbanos, y el derecho a la participación ciudadana y construcción de la ciudad.

En el caso de los asentamientos precarios, tal como plantea la Defensoría del Pueblo, los mismos presentan condiciones precarias de habitabilidad, sin acceso a los servicios básicos de saneamiento, es decir, agua potable, gas, electricidad, cloacas, disposición de los residuos sólidos; generando, en muchos casos, consecuencias negativas sobre la salud de la población. En lo que respecta a la construcción de las viviendas, suelen realizarse con materiales tales como cartón, chapa, bolsas, plásticos y tierra, lo que lleva a que presenten en muchos casos riesgos de derrumbe y desmoronamiento debido a la precariedad de sus estructuras (Defensoría del Pueblo, 2006). Las condiciones habitacionales en los asentamientos, por lo tanto, constituyen la manifestación extendida de pobreza, indigencia y vulnerabilidad social en la que sus habitantes se encuentran expuestos a múltiples riesgos y a situaciones de violencia urbana.

\section{LA INTERVENCIÓN DE POLÍTICAS URBANAS: CASO PROMEBA}

El desarrollo de políticas públicas que pudieran paliar estas problemáticas derivadas del crecimiento urbano debía ser, tal como plantean Ozslak y O’Donnell (1976), no una respuesta aislada sino, más bien, una serie de respuestas e iniciativas, que permitan determinar la posición del Estado en relación con las cuestiones que involucren a los distintos sectores significativos del conjunto social. En este sentido, las políticas habitacionales, cualquiera sea, deben poder garantizar el acceso a las personas a un lugar donde habitar, el poder accede al suelo en tanto medio habitable, con los acondicionamientos físicos, sociales y ambientales aptos para el desarrollo de condiciones de habitabilidad dignas en cualquier asentamiento humano (Trivelli, 1982).

A lo largo de las últimas décadas han surgido distintas generaciones de políticas de intervención urbana. La primera generación correspondía a políticas de vivienda. Surgidas en la década del 50, en un contexto de aumento de índices de pobreza urbana como resultado del creciente proceso de urbanización en América Latina. Estas políticas promovían reemplazar y erradicar los asentamientos precarios por nuevos conjuntos habitacionales. A pesar de su poca efectividad, dado al aumento de pobreza y asentamientos, y de los elevados costos que suponían, fueron sostenidas en función de intereses privados y 
políticos (Lentini, Palero,Montaña,Torres, De Rosas, Levatino, Diocondo, Riveira, 2007; Fernandéz Wagner, Varela, Silva, 2004).

La segunda generación de políticas fueron las habitacionales (o alternativas), que aparecieron en los años 70, frente al fracaso de los programas de erradicación. Esta nueva línea de programas no representaba un reemplazo de las de primera generación, sino que ambas se superponían. Las mismas apuntaban a los lotes, la garantía y servicios a partir de los cuales las familias pudiesen ir completando sus casas (Fernández Wagner et al., 2004).

Las politicas de tercera generación o políticas de facilitación surgen durante las décadas del ' 80 y del ' 90 , en un escenario de profunda demanda habitacional y aumento de carencias. En un contexto de economía neoliberal se modificó la focalización de las políticas, buscando con esto la asunción del Estado en su rol de facilitador del funcionamiento del mercado habitacional (Lentini et al., 2007).

La crítica a este modelo de políticas para el hábitat abrió el paso al desarrollo de programas alternativos, a partir de los cuales el Estado ofrecía una amplia gama de ofertas de servicios en tanto soluciones a la problemática habitacional. Es así como el problema de la regularización y urbanización de asentamientos precarios comienza a aparecer como tema central en elaboración de políticas y programas de intervención urbana (Canestraro, 2010). El objetivo de estos programas es generar el reconocimiento legal y jurídico de esas grandes áreas de la ciudad que se mantenían dentro de los estándares de la informalidad, la ilegalidad e irregularidad de la producción del espacio, así como apartadas y segregadas de la planificación urbana (Clichevsky, 2000; Marengo y Elorza, 2009).

Estas intervenciones exponen la influencia del economista liberal peruano Hernando De Soto (1986), quien supone que el puntapié inicial para promover el desarrollo económico en los países en transición se basa en el derecho jurídico sobre el suelo, por lo que la obtención de un título de propiedad basta para favorecer el acceso al crédito de los sectores populares, generando un impulso en sus iniciativas microempresariales, revitalizando el "capital muerto".

Esta propuesta fue fuertemente cuestionada por algunos investigadores quienes sostenían, entre otras cosas, que las entidades financieras, no crean líneas de crédito para los pobres dado su imposibilidad de devolverlo, a la vez que, el hecho de contar con un título de propiedad constituye una condición necesaria pero no suficiente para lograr el acceso al crédito (Relli Ugartamendía, 2009).

A pesar de las críticas, la teoría de De Soto ha tenido influencia en la producción de políticas de integración y regulación del suelo impulsadas por organismos internacionales tales como el Banco Interamericano de Desarrollo (BID), el Banco Mundial, UN-Habitat, PNUD, entre otras agencias, implementadas en varios países latinoamericanos (Canestraro, 2010). De esta forma, el hábitat informal urbano se convirtió en una línea prioritaria de intervención y financiamiento.

Las políticas de regulación impulsadas por los gobiernos, sobre todo municipales, tienden a solucionar dos tipos de problemas relacionados con la ilegalidad: las problemáticas de dominio, y aquellos que refieren a los aspectos urbano-ambientales. Un tercer objetivo se puede sumar, el focalizado en la inclusión social de los pobladores que 
habitan la ciudad de forma segregada, social y espacialmente (Clichevsky, 2003). En las últimas décadas, proliferaron programas integrales en términos de mejoramiento urbanoambiental e integración social, especialmente los que se promueven por medio de financiamiento externo, que han tenido un alcance limitado y que lejos de ser políticas preventivas han sido curativas e, incluso, al decir de Smolka (2003), han reforzado el círculo pobreza informalidad. Un claro ejemplo es el Programa de Mejoramiento de Barrios (ProMeBa) (Arqueros Mejíca y Canestraro; 2017).

Implementado en la Argentina desde el año 1997, el ProMeBa, es un programa nacional financiado a través de un crédito otorgado por el BID en conjunto con la Secretaría de Desarrollo Social de la Nación, cuya finalidad es contribuir a la inclusión urbana y social a partir de la legalización de la tenencia de la tierra, de la provisión de obras de infraestructura urbana, equipamiento comunitario, saneamiento ambiental, del apoyo a procesos de organización barrial y autogestión comunitaria, mejoras en las viviendas y entornos urbanos, combatiendo así a la pobreza, y mejorando las condiciones habitacionales de las familias en barrios marginales y asentamientos informales de zonas urbanas.

En lo que respecta a sus delimitaciones, el programa presenta una focalización social y espacial. El enfoque poblacional/social implica la identificación de las posibles familias susceptibles de ser consideradas como población beneficiada, en virtud de sus niveles de ingreso mínimo (deben ubicarse en el primer quintil de la población). El otro enfoque, el espacial, implica la selección y delimitación de un territorio a partir de sus condiciones de pobreza y precariedad urbana (Wagner et al., 2004).

En lo que respecta a su estructura organizativa, en la Argentina el ProMeBa se aplica a través de la Subsecretaría de Desarrollo Urbano y Vivienda, Secretaría de Obras Públicas, Ministerio de Planificación Federal, Inversión Pública y Servicios. Su ejecución se realiza por medio de la participación conjunta de las Unidades Ejecutoras Provinciales (UEP), los municipios, organizaciones sociales y empresas de servicios, organizándose en diversas etapas: ProMeBa I, ProMeBa II, ProMeBa III.

Actualmente, el ProMeBa está operando en 19 provincias del país a través de 649 proyectos (579 terminados, 45 en proceso y 25 en proceso de licitación) que benefician aproximadamente a unas 43.057 familias.

\section{EL PROMEBA EN MAR DEL PLATA}

En la ciudad de Mar del Plata, recién en el año 2010 comienza a materializarse la implementación del Programa de Mejoramiento de Barrios, con una inversión financiada por el BID cercana a los 170.000 .000 de pesos, siendo el récord de financiamiento internacional que ha recibido la ciudad. Dirigidos por la Secretaría de Obras y Planeamiento Urbano del Municipio y coordinado por su titular Guillermo de Paz (Unidad Ejecutora Municipal), el mismo se enfoca en lograr mejoras en la infraestructura pública, en la red vial y pluvial, la pavimentación y en lo que respecta la red de gas. También, se busca trabajar con parte de la población beneficiada, apostando a la participación de estos para el desenvolvimiento de cada proyecto y a partir del dictado de 
talleres y cursos de capacitación para facilitar la salida laboral de los vecinos de los barrios intervenidos.

El contexto en el que desarrolla este programa se dio en el marco de selección de Mar del Plata en la Iniciativa Ciudades Emergentes y Sostenibles (ICES), financiada también por el BID, la cual desde un enfoque integral e interdisciplinario busca organizar, identificar y priorizar intervenciones urbanas para alcanzar el crecimiento sostenible de las ciudades emergentes de América Latina y el Caribe. A nivel local, Mar del Plata fue la primera ciudad en ser escogida en Argentina para participar dadas sus características urbanas y el marco político-institucional propio del momento.

Los epicentros del Programa de Mejoramiento de Barrios en la ciudad son los barrios Parque Palermo y Las Heras (delimitado entre las calles San Cayetano, el Ferrocarril Nacional Gral. Roca, Gutemberg, Reforma Universitaria, Mario Bravo y Labarden), debido a la extrema vulnerabilidad urbana y social que ambos presentan. Estos barrios son considerados como zonas no integradas o regularizadas dentro de la estructura urbana de la ciudad, es decir, áreas segregadas socio-espacialmente del espacio urbano. Localizados al suroeste del periurbano marplatense, a dos $\mathrm{km}$ de distancia uno del otro, estos barrios se caracterizan por su alta densidad poblacional estimada en 4.763 personas, 1182 familias, 1517 viviendas (Censo ProMeBa 2011), la escasa disposición de servicios y equipamiento urbano (lo que genera condiciones habitacionales deficientes), altos niveles de vulneración de derechos y de violencia frente a la falta de seguridad policial y ausencia de los organismos del Estado.

\section{Gráfico 1}

Área de intervención del ProMeBa en Mar del Plata

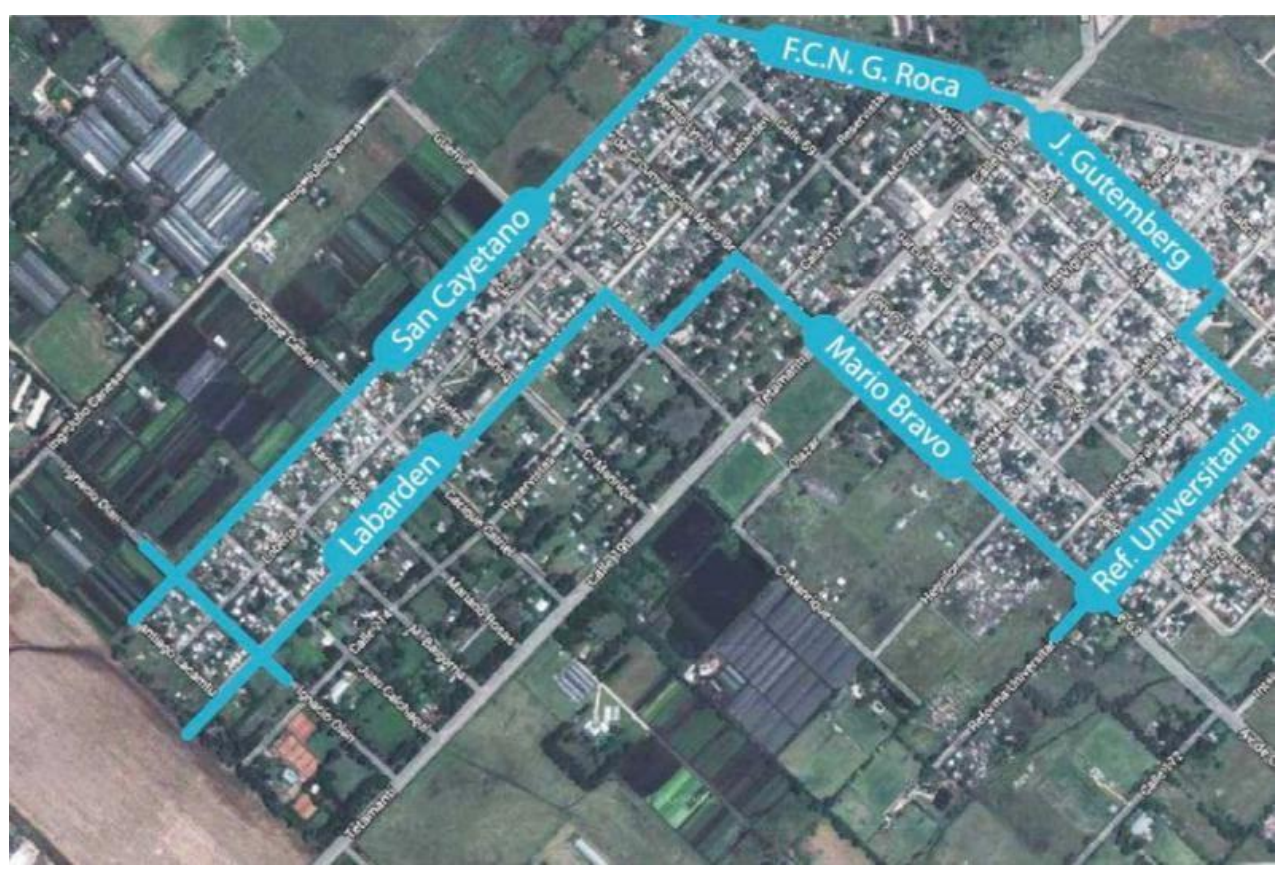

Fuente: (Municipalidad de General Pueyrredón, 2013)

De las 157 hectáreas que supone la superficie total del barrio Las Heras, el ProMeBa abarca únicamente 32 de esas hectáreas. En el caso de Parque Palermo, solo 44 de las 77 
hectáreas totales que lo conforman son intervenidas por el programa. No obstante, se estima que la situación de precarización habitacional y la cantidad de familias viviendo en la informalidad ha aumentado notablemente a partir del contexto pandémico por el COVID-19 y las medidas de Aislamiento Social, Preventivo y Obligatorio (ASPO).

Desde el 2010, año en el que se comienza a implementar el ProMeBa, ya se han realizado el proyecto de Infraestructura Pública (terminado), en la fase E1A, en la que se han destinado \$38.778.068,28; el proyecto de Red de Gas (terminado), en la fase E1B, con un costo de \$7.252.113,85; el proyecto de Pavimentación (terminado), en la fase E3, con un valor de proyecto de $\$ 139.673 .951,85$; y el proyecto de Red Vial y Pluvial (en ejecución) que ya lleva invertidos unos $\$ 59.908 .329,80$.

A pesar de todas estas obras y mejoras que incluyen tareas de alumbrado público, desagües pluviales, red eléctrica, de agua potable, red vial (cordón cuneta y carpeta asfáltica), sendas peatonales, red para la provisión de gas natural, la construcción de refugios para paradas de micros y la realización de talleres de capacitación técnica para la facilitación de la salida laboral y la mantención colectiva de los barrios, los reclamos de vecinas y vecinos de las zonas intervenidas aún siguen siendo constantes en lo que respecta la demanda de mejores condiciones habitacionales, las problemáticas de las continuas inundaciones, la falta de reconocimiento jurídico y la situación crítica respecto a la inseguridad en los barrios, dado a la negativa de la policía municipal de ingresar allí, situaciones que se han visto profundizadas y agravadas en el actual contexto pandémico.

Por otra parte, la "formalización" de estos barrios segregados lo que produce es una mejora de las condiciones habitacionales de las familias que en ellos habitan a la vez que se genera un aumento de los costos como resultado de las mejoras de infraestructura y servicios. En algunas ocasiones, esta situación supone, más que un beneficio, una problemática en los casos en que las familias no logran costear y solventar los nuevos costos que implica la formalización de esas áreas. Se genera así un círculo vicioso, donde los propios habitantes de los barrios intervenidos se ven impulsados a habitar nuevamente en la informalidad, reproduciendo así y mecanismos de segregación urbana.

\section{CONCLUSIÓN}

Como ya se ha mencionado, el derecho a la ciudad pronunciado por Henri Lefebvre (1968) implica el derecho de los habitantes urbanos a construir, decidir y crear la ciudad, y hacer de esta un espacio privilegiado de lucha anticapitalista, a partir de la constitución de una identidad urbana y la habilitación para la participación política. Sin embargo, en la actualidad, las ciudades lejos están de garantizar estos derechos, y es que el incremento de la urbanización trae aparejado la reproducción de mecanismos de segregación socioespacial, el empobrecimiento de la experiencia urbana, y nuevas formas de producción de hábitat precario.

En concordancia con la noción de habitar la ciudad, la problemática del derecho a la misma resurge con énfasis dentro del campo académico de los estudios urbanos, así como de los movimientos y movilizaciones sociales, impulsando el replanteamiento y la puesta en marcha de políticas de intervención urbana. Es que el derecho a un hábitat 
digno implica un análisis más integral y holístico, y no de corte reduccionista como suelen proponer las políticas urbanas de las últimas décadas.

El estudio de caso de Mar del Plata representa un ejemplo claro que nos permite analizar tanto el impacto que pueden tener las diversas intervenciones de enfoque urbano, como la urgencia de estas en la ciudad. Considerando que Mar del Plata es la ciudad del interior de la Provincia de Buenos Aires con más barrios precarios (en total hay alrededor de 80 barrios caracterizados como villas o asentamientos precarios) resulta llamativa la tardía implementación del ProMeBa en la ciudad.

Por otro lado, su implementación podría abrir una serie de interrogantes a desarrollar en futuras investigaciones: ¿Constituyen programas como el ProMeBa una respuesta sistematizada a las problemáticas urbanas sin considerar el contexto particular de cada ciudad, cada barrio o cada conjunto de familias en particular?, ¿Qué relevancia se le dio a la hora de elaborar un diagnóstico completo e integral a aspectos tales como la inseguridad, la violencia, y el desempleo?, ¿No constituyen estos aspectos variables a tener en consideración?, ¿En qué medida intervenciones urbanas como éstas logran garantizar el derecho participativo de la población?, ¿Se ha tenido en consideración las verdaderas demandas de las familias habitantes de barrios segregados socio-espacialmente?, ¿De qué manera se pueden desarrollar y aplicar políticas como el ProMeBa sin caer en un círculo vicioso en donde más que prevenir o reducir la pobreza y exclusión social, se profundicen debido al aumento de los nuevos costos de vida por la regulación urbana?

\section{REFERENCIAS BIOGRÁFICAS}

Arqueros Mejica, M.S, Canestraro, M.L. (2017), "Procesos sociales y dinámicas urbanas: debates sobre el abordaje de la informalidad”, Cardinalis, UNC, Argentina.

Bourdieu, P. (1997), "Rařnes prácticas”, Anagrama, Barcelona.

Brun, J. (1994), "Essai critique sur la notion de ségrégation et sur son usage en géographie In Jacques Brun \& Catherine Rhein”, La ségrétion Dans la ville, L’Harmattan, Paris.

Caprón, G. (2006), "Las escalas de la segregación y de la fragmentación urbana”, Cemca, México.

Canestraro, M.L. (2010), "Entre el hecho y el derecho. De actores y prácticas en tres procesos de producción de hábitat (Mar del Plata, 1983-2009)", Tesis Doctoral, Universidad de Buenos Aires.

Canestraro, M. L. (2013), “illegales, irregulares, informales...?: aportes para un debate sobre el acceso al suelo.", Nómadas, Revista Crítica de Ciencias Sociales y Jurídicas, volumen especial (América Latina), 249-270. Madrid: Universidad Complutense de Madrid.

Canestraro, M.L. (2016), "Sobre el derecho a la ciudad y el acceso al suelo urbano. Reflexiones a partir de intervenciones estatales recientes (Mar del Plata, 2012-2015)", ESTUDIOS SOCIOTERRITORIALES, Revista de Geografía, Argentina.

Canestraro, M.L; Elorza, A.L. (2019), “MEDIO AMBIENTE Y HABITAT”, CLACSO; Ciudad Autónoma de Buenos Aires: PISAC - Programa de Investigación sobre la Sociedad Argentina Contemporánea.

Censo ProMeBa (2011) 
Castells, M. (1974), "La cuestión urbana”, Siglo XXI Editores, España.

Centro de Estudios Legales y Sociales (2015), "Una perspectiva de derechos sobre las politicas de desarrollo y de acceso justo al hábitat", Derechos humanos en Argentina. Informe Anual, capítulo XI. Buenos Aires.

Clichevsky, N. (2000), "Informalidady segregación urbana en América Latina. Una aproximación", Publicación de las Naciones Unidas, Santiago de Chile.

Clichevsky, N. (2003), "Pobreza y acceso al suelo urbano: algunas interrogantes sobre las politicas de regulación en América Latina”, Naciones Unidas, Santiago de Chile.

Cravino, M. C. (1998), "Las organizaciones villeras en la Capital Federal entre 1989-1996. Entre la autonomía y el clientelismo", Ciudad Virtual de Antropología y Arqueología.

Defensoría del Pueblo de la ciudad de Buenos Aires (2006): "Informe de Situación. Desalojos de Nuevos Asentamientos Urbanos", Buenos Aires, disponible en Internet: http://www.defensoria.org.ar/institucional/doc/asentamientos.doc

De Soto, H. (1986), "El otro sendero", Instituto Libertad y Democracia, Lima.

Do Rio Caldeira, T. (2007), "Ciudad de muros", Barcelona: Gedisa.

Duhau, E. (2003), "La división social del espacio metropolitano en Las reglas del desorden”, Habitar la metrópoli, Siglo XXI, México.

Durand-Lasserve, A. (1997), Prólogo. En A. Azuela y T. François (Coords.) "El acceso de los pobres al suelo urbano (pp. 9-12)", México: CEMC-UNAM

Fernándes, E. (2003), "Perspectivas para a renovação das políticas de legalização de favelas no Brasip", En P. Abramo (Org.) A ciudade da informalidade. O desafio das cidades latinoamericanas. Rio de Janeiro: Livraria Sette Letras, FAPERJ.

Fernández Wagner, R. Wagner, R.F; Varela, O.; Silva, M.R, (2004), “La complejidad ausente en los programas de intervención socio-espacial inscriptos en el paradigma de la reducción de la pobreza. El caso del PROMEBA en el Gran Buenos Aires.", II Congreso Nacional de Políticas Sociales, Mendoza.

Kessler, G. (2014), “Controversias sobre la desigualdad”, Argentina, 2003-2013. Buenos Aires: Fondo de Cultura Económica.

Lefebvre, H. (1968), “El derecho a la ciudad”, 3ra. ed. Barcelona: Península.

Lentini, M., Palero, D., Montaña, D., Torres, L., De Rosas, F., Levatino, B., Diocondo, J., Riveira, V. (2007), “Alternativas para el hábitat popular en Mendoza: El Programa de Mejoramiento de Barrios (PROMEBA) como respuesta a la pobreza urbana", Recuperado el 20 de marzo de 2014 de: http://bdigital.uncu.edu.ar/objetos_digitales/2811/ lentinihabitat2.pdf.

Marengo, C. y Elorza, A. L. (2009), "Globalización y politicas urbanas. La politica habitacional focalizada como estrategia para atenuar condiciones de pobreza urbana: los programas implementados en Córdoba y los desafíos pendientes", En: Revista Cuaderno Urbano, Chaco, Argentina. 
Massey, D. (1996), "The Age of Extremes: Concentrated Affluence and Poverty in the TwentyFirst Century", Springer on behalf of the Population Association of America.

Merklen D. (1991), "Asentamientos en La Matanza. La terquedad de lo nuestro". Buenos Aires: Catálogos Editora.

Municipalidad de General Pueyrredón (2013), recuperado de: https://www.mardelplata. gob.ar/documentos/gobierno/promeba\%20-\%20las\%20heras.pdf

Notcheff, H. (1999), "La politica económica en la Argentina de los noventa. Una mirada de conjunto", Revista Época (1), p. 22.

O’Donnell, G.; Oszlak, O. (1976), "Estado y Politicas Estatales en América Latina", Documento de Estudios CEDES. Buenos Aires.

Oszlak, O. (1983), "Los sectores populares y el derecho al espacio urbano", En: Revista de la Sociedad Central de Arquitectos, Buenos Aires, Argentina.

Pírez, P. (1995), “Actores sociales y gestión de la ciudad”, Ciudades, México.

Relli Ugartemendía, M. (2009), "Regularización de la informalidad urbana: notas para el estudio de las acciones emprendidas en la provincia de Buenos Aires", Argentina. XII Encuentro de Geógrafos de América Latina, Montevideo, Uruguay.

Rodríguez, M. F. (2009), "Notas sobre los conceptos de los "Nuevos Asentamientos Urbanos" (NAUs) en la ciudad de Buenos Aires," Revista Pampa, Año 5, N5.

Scarponetti, P. (2019), "Estudios sobre condiciones de vida en la Argentina contemporánea," CLACSO; Ciudad Autónoma de Buenos Aires: PISAC.

Smolka, M. (2003), "A regularização da ocupação do solo urbano: a solução que éparte do problema, o problema que é parte da solução", En P. Abramo (Org.) A cidade da informalidade. O desafio das cidades latino-americanas. Rio de Janeiro: Livraria Sette Letras, FAPERJ.

Topalov, C. (1979), "La urbanización capitalista: algunos elementos para su análisis", Edicol, México.

Trivelli, P. (1982), "Accesibilidad al suelo urbano y la vivienda por parte de los sectores de menos ingresos en América Latina", Revista EURE, Chile.

Velázquez, G. (2007), "Población, territorio y calidad de vida", en Torrado Susana (comp.), Una historia social del siglo XX, Tomo II, Buenos Aires, Edhasa.

\section{CITAR COMO:}

Sanchez Bozo, C. B., Isea, E., \& Vera, E. (2021). Realidad de la práctica pedagógica en tiempos de pandemia. Puriq, 3(4), 491-502. https://doi.org/10.37073/puriq.3.4.188 